\title{
Experiencia de prevención escolar de las drogas en Argentina basado en la teoría de Pichon Riviere
}

\author{
Milán, T.*; Calderoni, A.**; Ressia, I. ***; RuIz, M.**** \\ Universidad Nacional de San Luis. Argentina. \\ * Prof. Titular de Psicopatología de la Licenciatura en Psicología, Directora del Proyecto de Investigación sobre Drogadicción. Facultad de Ciencias Humanas \\ * Prof. Adjunta de Toxicología de la Licenciatura en Bioquímica. Facultad de Química, Bioquímica y Farmacia \\ ** Jefe de Trabajos Prácticos de Metodología de la Investigación de la Licenciatura en Psicología. Facultad de Ciencias Humanas \\ ****Pasante en el Proyecto de Investigación sobre Drogadicción.

\section{RESUMEN}

Se aplicó un programa de prevención de la drogadicción en el medio escolar cuyo objetivo fue generar un espacio de diálogo con los adolescentes para discutir las temáticas propias de su edad, detectar el nivel de prevalencia de la problemática de las drogas y el conocimiento que poseían acerca de las mismas. La hipótesis es que este conocimiento genera pautas de acción frente al ofrecimiento de drogas.

Se trabajó con estudiantes de nivel medio de dos escuelas públicas de San Luis (Argentina), utilizando un encuadre grupal según el ECRO desarrollado por Pichon-Rivière y se aplicaron otras técnicas grupales.

La utilización de esta metodología permitió que los adolescentes hablaran desde su experiencia personal y surgieran dudas, confusiones y preguntas acerca de las drogas. La coordinación intervino brindando información y aclarando confusiones, contribuyendo a que los adolescentes pudieran construir un modelo para operar en la vida cotidiana donde están las drogas y los riesgos.

Palabras claves: Prevención escolar, drogas, técnicas grupales, Argentina.

\section{ABSTRACT}

A program for drug prevention was applied in the school setting with the aim of generating an adequate instance to favor interaction with teen agers. In that sense, topics connected to adolescents were discussed in order to detect the level of prevalence given to drugs as well as their knowledge about drug abuse. The hypothesis is that information will generate actions on the part of the students when drug is offered.

The experiment was carried out with high school students from two public schools in San Luis. ECRO groups frame developed by Pichon-Rivière as well as other group techniques were applied.

This methodology permitted students to talk from their own personal experience allowing doubts, misconceptions and questions about drugs to arise. The necessary information to clarify these issues was provided by the coordination of the experiment, helping students to build up a solid standpoint from where to deal with the risks of drug in their daily lives.

Key words: school prevention, drug use, adolescence, group technics, Argentina.

\section{INTRODUCCION}

$\mathbf{E}$ ste trabajo expone una experiencia de prevención de la drogadicción en dos escuelas estatales del nivel medio en la ciudad de San Luis (Argentina).

Las circunstancias vitales que llevan a una persona a optar por las drogas son anteriores al consumo. La prevención debe actuar sobre los factores de riesgo y protección que subyacen a los comportamientos adictivos (Calafat, 1998). Debido a las características propias de su etapa evolutiva, los adolescentes se constituyen en uno de los principales grupos de riesgo para el consumo de drogas (Dumoulin, P. y otros, 1998). En ellos, la institución escolar ocupa un lugar de relevancia en el desarrollo de acciones tendientes al desenvolvimiento personal y la promoción de la salud. La escuela es un agente de socialización que interviene en una fase del proceso de maduración donde la presencia del adulto tiene fundamental incidencia.

Herrero Yuste, M. (1999) afirma que los programas de prevención dirigidos al ámbito escolar deben integrar la participación de los docentes, alumnos y padres en un marco comunitario que dé coherencia a los mensajes y promuevan acciones complementarias en una unidad de objetivos. El fenómeno del abuso de drogas no es un problema susceptible de ser reducido 
a una cuestión de sustancias, sino que depende de la interacción del sujeto, del medio y de la droga (Pons Diez, J.; Pinazo Hernandis, S.1997). Es importante también que las campañas de prevención estén apoyadas sobre investigaciones epidemiológicas y respondan a una planificación sostenida en el tiempo.

El programa de prevención aplicado perseguía como objetivo general generar un espacio de diálogo con el grupo de adolescentes para que ellos pudieran exponer la problemática propia de su edad. Como objetivos específicos el programa buscaba detectar el nivel de prevalencia de la problemática de la drogadicción en los adolescentes y el conocimiento que poseían acerca de las drogas. El lema del programa es "Conocer para comprender, comprender para prevenir". Partimos de la hipótesis que este conocimiento genera pautas de acción frente al consumo de tóxicos. Esta hipótesis se fundamenta en el modelo teórico de Pichon-Rivière acerca del Esquema Conceptual Referencial y Operativo (ECRO). El ECRO es un sistema organizado de conceptos referidos a un sector de la realidad: los procesos interaccionales, el interjuego o dialéctica entre sujetos; es un modelo para operar sobre la realidad y además un repertorio teórico técnico. El Esquema Conceptual sería un conjunto organizado de conceptos que se encuentra en permanente construcción, abierto a la posibilidad creativa de cada uno de los integrantes en la prevención para recrearlo desde múltiples experiencias. EI ECRO es referencial porque alude al campo concreto en el que se va a trabajar; y es operativo porque sirve para promover una adaptación activa a la realidad. Es un modelo útil para la investigación- acción.

Pichon-Rivière considera que la comunicación es el riel del aprendizaje; para poder intercambiar mensajes cada vez mas ricos los interlocutores deben poseer un código en común o deben construirlo. La construcción de este marco referencial común es un proceso a realizar a partir del conocimiento que el adolescente posee y abierto al cambio provocado por la etapa evolutiva. La utilización de este marco teórico y metodológico proveerá a los destinatarios del programa de un modelo transferible a la vida cotidiana en la comunidad donde está la droga y donde existen los riesgos.

El adolescente establece un vínculo particular con el conocimiento. Siguiendo a Bion, Meltzer discrimina dos tipos de conocimiento: 1) el conocimiento de las cosas que vienen directamente de la autoridad; 2) el que surge de la experiencia y que implica la tolerancia a la angustia y confusión que emerge en el establecimiento de un vínculo emocional con el mundo. Meltzer, D. (1978) identifica cuatro diferentes comunidades dentro de las cuales el adolescente se encuentra en constante movimiento: el ir hacia atrás hasta volverse niño, el ir hacia adelante para ser adulto, el estar en el mundo de los adolescentes o sentirse muy aislado, separado y grandioso. En este proceso, la emergencia del conflicto dependencia - independencia se transforma en un facilitador del acceso a las drogas, las que pueden ser visualizadas ilusoriamente como vehículo para oponerse a la "cultura de los adultos" y definir su pertenencia a un grupo.

En Argentina, a través de los medios masivos de comunicación se han llevado a cabo múltiples campañas de prevención de la drogadicción pero no contamos con una evaluación de sus resultados. Es fundamental evaluar las campañas de prevención y adecuar los programas a las condiciones sociales y culturales (Calafat, A. 1998). La vasta mayoría de los programas de prevención son evaluados después que los chicos han dejado la escuela, de manera tal que no es posible garantizar la eficacia de los mismos para los adolescentes en riesgo (Vitaro, F., Maliantovitch, K., et al, 1998)

\section{MATERIAL Y METODO}

\section{Muestra}

Se trabajó con una muestra de tipo accidental, integrada por 92 alumnos entre los 16 y los 19 años de edad, de ambos sexos, pertenecientes a cuatro divisiones de $5^{\circ}$ año de dos escuelas estatales, del nivel medio de la ciudad de San Luis. Una de ellas de nivel socio-económico-cultural medio bajo homogéneo y la otra con características más heterogéneas ya que alberga sujetos de diferentes niveles socioeconómicos y culturales pero con preponderancia de clase media.

\section{Procedimiento e instrumentos}

El trabajo se realizó con un encuadre grupal según el ECRO elaborado por Enrique Pichon-Rivière (1993). En cada uno de los grupos, el coordinador comienza la tarea dando a conocer sus objetivos, lo que ubica a los adolescentes como protagonistas del trabajo a realizar. Además, se emplearon otras técnicas auxiliares:

- Técnica de presentación cruzada: es una técnica de iniciación de una actividad grupal en la que se pone en juego la representación que el otro tiene de sí para facilitar la constitución del grupo y el vínculo con el equipo de coordinación. La consigna es: "nadie se presenta a sí mismo", cada participante del grupo presenta a un compañero.

- Frases incompletas: consiste en completar por escrito, en forma individual y anónimamente una serie de frases incompletas que fueron elaboradas ad hoc a fin de obtener información acerca de: el sentimiento de identidad; vivencia de continuidad 
temporal; incidencia de personas significativas (pares, padres, adultos) ; conflicto (conductas predominantes ante situaciones de elección); imagen de la sociedad, la cultura y los valores; el nivel de prevalencia de las adicciones.

- Cuestionario: Este instrumento se ha utilizado con los mismos propósitos desde 1995 en escuelas públicas y privadas de la ciudad de San Luis. Consta de ocho preguntas, de las cuales solo una presenta alternativas múltiples. Las mismas recogen información acerca de : la actitud frente al ofrecimiento de drogas, actitud frente al sujeto que usa drogas, conocimiento acerca de las drogas y sus efectos, los mensajes e información recibidos a través de la televisión, los comentarios respecto a ellos, los interlocutores válidos para hablar del tema de las drogas (alternativas múltiples) y el recuerdo de esas conversaciones.

Los objetivos de su aplicación fueron posibilitar el registro del conocimiento y las actitudes que los adolescentes tenían sobre las drogas y servir de disparadores para la discusión grupal.

Luego de la aplicación de cada una de estas técnicas, se realizó una discusión grupal durante la que se pudo corroborar, rectificar o ampliar datos y recoger opiniones y fantasías observando las tendencias dominantes del grupo.

\section{RESULTADOS}

A partir de un análisis cuantitativo y cualitativo de los datos se presentan los siguientes resultados:

\section{Técnica de la Presentación Cruzada.}

Los participantes destacaron datos personales y se atribuyeron cualidades propias de la comunidad adolescente (la recreación, el deporte, amistad, la sexualidad). La mayoría se refirió a características que los definían como pertenecientes a distintos subgrupos e involucrándose en la descripción. Es importante destacar que algunos sujetos identificaron a sus compañeros con rasgos ligados al consumo de sustancias: "le gusta tomar alcohol...", "hace gimnasia con el faso ${ }^{2}$..."

\section{Frases Incompletas.}

En este trabajo solo se presentarán los resultados de aquellas frases en las cuales los adolescentes aludieron espontáneamente a la drogadicción.

Expresaron que la droga es una de las amenazas a las que debe enfrentarse en esta etapa de búsqueda de la identidad: "Lo más difícil a esta edad es llegar a ser sí mismo y las drogas".

Cuando opinaron acerca de los adultos, se pudo observar claramente el funcionamiento del mecanismo de adjudicación y asunción de roles. Algunos consideraron que los adultos los visualizan como los depositarios de todos los aspectos negativos de la sociedad, adjudicándoles el papel de "inadaptados", "descontrolados", que "consumen drogas, alcohol", aspectos que se resumen en la siguiente frase: "Los adultos piensan que los jóvenes son la sociedad perdida". En contraposición a lo anterior, cuando emitieron su opinión sobre los jóvenes afirmaron: "son el futuro", "son los únicos que pueden hacer cambiar el país", convirtiéndose en depositarios de los aspectos positivos e idealizados, esto es, tener confianza en su capacidad potencial para hacer frente a las diversas situaciones que se le presentan y poder resolverlas de modo adecuado. En contraste con esta idealización de la juventud, algunos se identificaron con la imagen que creen que los adultos tienen de ellos: "somos descontrolados", "nos gusta vivir de fiesta en fiesta". Otros se identificaron con los adultos, como no pertenecientes a la comunidad adolescente, y dijeron que los demás: "son imprudentes, no se cuidan de ningún peligro", "son así por culpa de la droga y el gobierno que está permitiendo ésto".

Los adolescentes les asignan toda la responsabilidad de las características negativas que se manifiestan en la juventud a la sociedad y al gobierno, es decir, a los adultos. Consideran que la existencia de la pobreza, la corrupción, la inseguridad, la injusticia, son factores que posibilitan y conducen al establecimiento de la violencia, el alcoholismo y la drogadicción. La imagen que tienen de la sociedad en general se expresa en estas frases: "La sociedad en la que vivo es una porquería, robos, muertes, droga, corrupción"; "Lo que más me preocupa de San Luis es el alcoholismo y la drogadicción". Esta crítica a la sociedad los identifica como pertenecientes a la comunidad adolescente e implicaría una resistencia a ingresar al mundo adulto.

\section{Cuestionario.}

Se elaboraron categorías a partir de las respuestas de los sujetos. No se observaron diferencias en las respuestas según sexo, edad y entre los adolescentes de una y otra escuela, de modo que los datos refieren a la totalidad de la muestra.

El análisis se realizó en función de tres ejes: a) la actitud frente a la droga y al sujeto que se droga, b) el conocimiento de los tipos de droga y los efectos que éstas producen, c) la información emitida por otros y la valoración de los mensajes recibidos.

2) Término del lunfardo utilizado habitualmente por los adolescentes para designar el cigarrillo de tabaco o de marihuana. 


\section{A) La actitud frente a la droga y al sujeto que se droga.}

El 96\% de los adolescentes afirmaron que frente al ofrecimiento de drogas no las aceptarían; muy pocos reconocieron que el ofrecimiento los colocaría en una situación de conflicto ante el cual no sabrían qué responder, ya que "dependería del momento" por el cual están atravesando; "del tipo de droga", o bien porque en ocasiones suelen consumir marihuana. Del total de los adolescentes encuestados, el 4\% reconoce haber consumido alguna droga ilegal.

Las razones dadas por quienes afirmaron que frente al ofrecimiento de drogas no las aceptarían fueron: que ocasionaría un daño o perjuicio a su vida, a su salud o al trato con familiares y amigos; que pueden prescindir de ellas; o que "son malas", expresando un juicio valorativo. Otros evidenciaron una actitud de huida y temor: "le digo que no y me voy", "no aceptaría por miedo a que me gusten". Muy pocos dieron como razón la información que poseen, aunque no especificaron el tipo de información o lo vincularon a la enfermedad y a la adicción, bajo la creencia de que el mero consumo de drogas lleva necesariamente a la adicción. También un escaso número dijo que no aceptarían drogas aludiendo a una experiencia indirecta o directa con ellas.

La mayoría de los adolescentes afirmaron que no aceptaría el ofrecimiento de drogas pero las razones dadas para sostener esta actitud están vinculadas a una información inespecífica y confusa. Otros no dieron razones por las cuales no aceptarían tal ofrecimiento.

Respecto a la actitud que tomaría frente a un familiar o amigo que consume drogas, la mayoría afirmó que lo ayudaría tomando una actitud activa. Otros reconocen su deseo de ayudar, pero no sabrían cómo hacerlo. Algunos manifestaron su deseo de ayudar, pero dudan de la efectividad de la ayuda debido a la percepción que tienen acerca del consumidor de drogas, como alguien que tiende a rechazar un pensamiento diferente al suyo y anula la posibilidad de ser ayudado. Es importante destacar que entre las respuestas que manifiestan el deseo de ayudar a quien consume, no existen diferencias entre quienes mostraron una actitud negativa frente al ofrecimiento y quienes reconocen haber consumido en alguna oportunidad.

B) El conocimiento acerca de los tipos de drogas y los efectos que éstas producen.

Ante la pregunta del tipo de drogas que conoce, la mayoría mencionó drogas ilegales (marihuana, cocaína, heroína, éxtasis). Algunos nombraron drogas legales como pegamentos, tabaco, alcohol y medicamentos. Con respecto a los efectos que producen las drogas, los adolescentes mencionaron y describieron una multiplicidad de ellos sin distinguir el tipo de droga/s que los provoca. La mayoría de los efectos reconocidos se asociaron a sensaciones displacenteras y a consecuencias negativas (violencia y pérdida de control, alteración del sistema nervioso, alucinaciones, muerte y dificultades sexuales, adicción). Muy pocos mencionaron efectos placenteros.

C) La información emitida por otros y la valoración de los mensajes recibidos.

Se les pidió a los sujetos que mencionaran tres mensajes o informaciones televisivos referidos a la droga, a fin conocer como los adolescentes resignifican los mensajes que "salen de la pantalla". La mayoría sólo pudo recordar un solo mensaje y el resto no recordó ningún programa televisivo o campaña que se refiera a este tema. Entre los que recordaron un solo mensaje, la mayoría mencionó una campaña organizada por el gobierno nacional, la de UNICEF, o algún programa que eventualmente hizo referencia a la drogadicción. Algunos a pesar de que no mencionaron un programa en especial, recordaron el contenido del mensaje televisivo de Miroli a modo de cliché ("no te drogués", "las drogas alteran el sistema nervioso, la sexualidad y la vida") .

Respecto a los comentarios que estos programas les merecen, la mayoría los valoró como buenos, les adjudicaron un valor educativo y preventivo. Pocos los evaluaron como regulares o insuficientes y otros no realizaron ningún comentario.

Indagados respecto a quiénes recurren en caso de dudas o curiosidades en relación a las drogas, la mayoría afirmó apelar a algún par (amigos, compañeros de la escuela), algunos a los padres y pocos a profesores o profesionales. La mayoría dijo que estas conversaciones aludían a consejos o informaciones, algunos no recuerdan nada y otros afirmaron que no han tenido este tipo de conversaciones.

\section{Discusión grupal posterior.}

Todos los adolescentes, espontáneamente, hablaron de las causas que llevan a una persona a recurrir al consumo de drogas; en primer término la presencia de un problema en el que el entorno familiar juega un papel fundamental, o la simple curiosidad por los efectos de los tóxicos.

Se observó en todos los grupos la referencia al alcohol y la discusión en torno a esta sustancia con mayor frecuencia que en relación a las drogas ilegales, inversamente a lo ocurrido en el cuestionario. Pusieron énfasis en no incluir el alcohol dentro de lo que ellos consideran como drogas y se originaron una serie de confusiones, por ejemplo: si el alcohol genera o no

3) La tira televisiva combina personajes de la realidad y personajes animados entre los que se destaca Fleco y Male; Fleco representa a un adolescente que se ve tentado a tomar drogas; el personaje real es un médico, Miroli, quien explica los daños que produce la droga. 
adicción, si quien bebe vino con las comidas puede considerarse un adicto, si el alcohol mezclado con gaseosas tiene mayor poder para producir efectos de alcoholización al igual que la mezcla de diferentes bebidas alcohólicas. Se mencionó también el medicamento y el riesgo adictivo de su uso se ilustró con referencia a situaciones de consumo familiar. Todos estos aspectos llevaron al grupo de coordinación a proveer información para aclarar las confusiones entre drogas legales e ilegales, entre efectos, uso, abuso y dependencia. A partir de la información obtenida de los coordinadores, los adolescentes intentaron armar argumentos para autorizarse a sí mismos el consumo del alcohol y aliviar cualquier temor y/o culpa. De este modo disociaron el contenido de la información y negaron los efectos o las consecuencias de la ingesta tóxica producidos por el alcohol, amparados en la legalidad de la sustancia y en la frecuencia de la práctica cultural.

Los temas que despertaron mayor interés fueron los efectos de las drogas en general, la relación entre violencia, droga y embarazo, los medicamentos, los adelgazantes y anabólicos. También surgió la idea "la droga es adicción", aunque en la producción escrita la noción de adicción aparece escasamente .

Del trabajo grupal surge que la posición axiológica "las drogas son malas" no es un juicio de atribución consistente porque no parece ser fruto de una reflexión personal sino la reproducción automática de una razón aprendida.

En la discusión grupal los adolescentes hablaron desde la experiencia personal y afirmaron que se drogan aquellos que integran grupos diferentes a los de ellos, "los hippies". Desde el punto de vista grupal la ilusión que funda al grupo y que sustenta su permanencia se asienta en la negación de la diferencia, de ahí que aquello que es diferente es puesto afuera y no es parte del grupo (Anzieu, 1978).

Durante el trabajo grupal los adolescentes, a través de sus portavoces, oscilaron entre dos posturas. Por un lado, el conocimiento respecto a las drogas y sus implicancias, el haber vivido alguna experiencia como protagonista o como espectador y por otro lado, el desconocimiento y distanciamiento respecto al tema. Estas diferencias guardan relación con la población de las escuelas. Los dos grupos de la escuela identificada como de un nivel socioeconómico medio bajo se dispusieron abiertamente al diálogo, solicitando información respecto a diferentes temas. Los adolescentes de la otra escuela (aquella identificada como de nivel socioeconómico más heterogéneo con preponderancia de clase media), si bien dejaron traslucir confusiones como las descriptas, adoptaron una actitud general de exhibir la información que tenían y cerraron el espacio para el intercambio con una actitud omnipotente de saberlo todo.

Las respuestas "No Se" a varias preguntas del cuestionario y que no condicen con el "nosotros sabemos todo esto" del posterior trabajo grupal, es una respuesta evasiva frente al compromiso que implica comprometerse y exponer el conocimiento del grupo de pares. Equivale a alejar del campo de la conciencia el problema y con él la fuente de ansiedad que genera.

Los padres se consignaron como uno de los principales interlocutores para hablar de dudas e interés por el tema de las drogas, pero contrariamente no recuerdan las conversaciones mantenidas con ellos. Esto hace dudar acerca del espacio para el diálogo y el intercambio entre padres e hijos. Los padres aparecerían como objetos preservados e idealizados, pero con quienes mantienen cierta distancia para poder permanecer en el grupo de los adolescentes y así poder construir un conocimiento propio acerca de las cosas que no provenga directamente de la autoridad, sino de la experiencia y del establecimiento de un vínculo emocional con el mundo.

En lo que respecta al recuerdo de las campañas de prevención, en la discusión grupal manifestaron expresiones peyorativas ("lo que decía el viejo ese") y de cierta burla por la inclusión de los personajes de ficción con quienes no se identificaban ("Ios ridículos dibujitos").

\section{CONCLUSIONES}

La experiencia contribuyó a generar un espacio de diálogo con los adolescentes donde la problemática del consumo de tóxicos se debatió ampliamente. El lenguaje utilizado por los adolescentes incluía términos relacionados con sustancias tóxicas y las diversiones nocturnas para describir aspectos de la identidad de los integrantes y como elemento que definía la pertenencia al grupo de pares. Este aspecto pone de relieve al consumo como organizador social y su influencia para una cultura adictiva. Los adolescentes consideran que la problemática de la drogadicción es testimonio de un sufrimiento psicosocial que es a la vez la expresión de una crisis social del país.

El conocimiento -cuantitativo y cualitativo- que poseen los adolescentes acerca de las drogas es escaso e inconsistente y desconocen la sobredeterminación de factores que intervienen en la constitución del consumo. La relación efecto /droga no aparece en forma clara, los efectos o bien son descriptos globalmente sin referencia a las drogas mencionadas, o no concuerdan con las sustancias. En general existe confusión entre los conceptos de droga y de adicción.

A medida que el diálogo grupal se fue fortaleciendo y se estableció un adecuado nivel de comunicación, emergieron preguntas, confusiones y errores acerca de un tema que consideran difícil e interesante. El trabajo grupal permitió el análisis de las contra- 
dicciones expresadas por los adolescentes que tendieron a llevar a la tarea grupal a una estéril situación dilemática, que funcionaba como defensa ante la situación de cambio. La tarea grupal consiste en la elaboración de las ansiedades producidas por el miedo a la pérdida de las referencias existentes y el miedo al ataque por la situación de pensar. Es esta la trayectoria para que el grupo pueda configurar un esquema conceptual, referencial y operativo. Creemos que la utilización de esta metodología dió como resultado una mayor participación de los adolescentes en el grupo, mayor compromiso emocional para hacer críticas, dar opiniones y demandar nuevas informaciones.

Respecto a las campañas de difusión en prevención de la drogadicción por los medios masivos de comunicación, nuestra investigación mostró que los adolescentes manifiestan rechazo por ellas porque las viven como una intrusión. El riesgo de esta actitud es que se banalice cualquier otra información valiosa independientemente de la fuente, incluida la difusión del conocimiento en el ámbito educativo.

\section{BIBLIOGRAFIA}

Anzieu, D. (1978) El grupo y el inconsciente. Madrid, Biblioteca Nueva.
Calafat, A. Hacia una prevención institucionalizada y científica. Adicciones 1998; 10/4: 291-297.

Dumoulin, P, Panunzi-Roger, N, Giot, MP, et al. (1998). Les dispositifs de prévention et le traitement des toxicomanies. Psychotropes Revue Internationale des Toxicomanie; 4/1: 9-30.

García-Señorán, M.; González González, S. (1998) Análisis y valoración de los métodos utilizados en la prevención del abuso de drogas en la adolescencia desde una orientación informativa y psicosocial. Adicciones; 10/2: 139-150.

Herrero Yuste, $M^{a}$ N, (1999) La intervención en drogadependencias desde el ámbito Municipal. Adicciones; 11/2: 87-90.

Meltzer, D. (1978) Seminarios de Novara, Quaderni di Psicoterapia Infantile, Roma, Editorial Borla.

Pichon-Rivière, E. (1993) El Proceso Grupal. Buenos Aires, Nueva Visión.

Pichon-Rivière, E. (1988) Del Psicoanálisis a la Psicología Social. Buenos Aires, Nueva Visión.

Pons Diez, J.; Pinazo Hernandis, S. (1997) Actitudes hacia las Drogas y Hábitat geográfico en una muestra de adolescentes. Adicciones; 9/2: 223 - 233.

Vitaro, F, Maliantovitch K, Bouchard C, et al. (1998) Prévention de la Consommation de Psychotropes Programme de prévention de la consommation de psycotropes chez les jeunes: suivi à moyen terme. Psychotropes Revue Internationale des Toxicomanie; 4/3: 111-131. 